\title{
Optimal Control Approach for Solving Linear Volterra Integral Equations
}

\author{
Sohrab Effati \\ Faculty of Mathematical Sciences, Ferdowsi University of Mashhad, Mashhad, Iran \\ E-mail: s-effati@um.ac.ir \\ Mohammad Hadi Noori Skandari \\ Faculty of Mathematical Sciences, Ferdowsi University of Mashhad, Mashhad, Iran \\ E-mail: hadinoori344@yahoo.com
}

\begin{abstract}
In this paper we present a new approach for linear Volterra integral equations that is based on optimal control theory. Some optimal control problems corresponding Volterra integral equation be introduced which we solve these problems by discretization methods and linear programming approaches. Finally, some examples are given to show the efficiency of approach.
\end{abstract}

Index Terms - Volterra integral equations, Optimal control, Linear programming.

\section{INTRO DUC TION}

Volterra integral equations arise in many physical applications, e.g., potential theory and Dirichlet problems and electrostatics. Also, Volterra integral equations are applied in the biology, chemistry, engineering, mathematical problems of radiation equilibrium, the particle transport problems of astrophysics and reactor theory, and radiation heat transfer problems $[1,2,3,4,5]$.

There exist the some valid approximate and numerical methods for solving Volterra integral equation such as Adomian decomposition method [6], Walsh functions method and multigrid approach [7, 8], Bernstein Polynomials method [9], collocation method $[10,11,12,13,14,15]$, Langrange interpolation method [16] Taylor-series expansion method [17,18,19], classical Neumann-series method [20], spectral methods $[21,22,23,24,25,26,27]$, finite element method [28], Sinc method [29,30], Galerkin method [31,32], wavelet approach [33], block-by-block method [34] and homotopy analytic method [35,36,37,38,39].

In this paper, we present a different approach from above methods for solving linear volterra integral equations which is based on the optimal control theory [40,41,42]. Consider the following linear Volterra integral equations of second kind:

$y(x)=\varphi(x)+\int_{a}^{x} g(x, t) y(t) d t, \quad x \in[a, b]$,

where $a$ and $b$ are constant, functions $g(.,$.$) and \varphi($. are continuously differentiable with respect to $x$. In equation (1), functions $\varphi($.$) and g(.,$.$) are known and$ $y($.$) is an unknown function. We assume that equation$ (1) have a solution.

The structure of this paper is as follows: Section 2 shows that solving Volterra integral equation (1) is equivalent to solve several optimal control problems. In section 3 , a discretization method is applied to convert the proble $m$ to the corresponding linear programming problem. In section 4, the applicability of the approach is illustrated in several examples in which the computed results are compared with the exact solution. Section 5, gives the conclusion of this paper.

\section{Optimal control problems}

In this section, we are going to introduce some optimal control problems corresponding Volterra integral equation (1). Let $p=\frac{\partial g}{\partial x}$. By using Leibnitz rule for derivatives we have:

$$
\begin{aligned}
\frac{d}{d x} \int_{a}^{x} g(x, t) y(t) d t & =g(x, x) y(x) \\
& +\int_{a}^{x} p(x, t) y(t) d t .
\end{aligned}
$$

Also from equation (2) and differentiating both sides of (1) respect to $x$ we have:

$$
\begin{aligned}
y^{\prime}(x)= & \varphi^{\prime}(x)+g(x, x) y(x) \\
& +\int_{a}^{x} p(x, t) y(t) d t, \quad x \in[a, b],
\end{aligned}
$$

Now let $x \in[a, b]$ be an arbitrary given number. We define the following problem:

$$
\left\{\begin{array}{l}
y^{\prime}(z)=\varphi^{\prime}(z)+g(z, z) y(z)+v(z), \quad z \in[a, x] \\
\int_{a}^{z}\left(v^{\prime}(t)-p(z, t) y(t)\right) d t=0, \quad z \in[a, x] \\
y(a)=\varphi(a), v(a)=0
\end{array}\right.
$$

Theorem II.1: Let $x \in[a, b]$ be an arbitrary number and $\left(y^{*}(),. v^{*}().\right)$ be solution of problem (4)-(6). Then we have:

$$
y^{*}(x)=\varphi(x)+\int_{a}^{x} g(x, t) y^{*}(t) d t
$$

Proof: Let $x \in[a, b]$. By initial conditions (6) and equation (5), we obtain

$$
v^{*}(z)=\int_{a}^{z} p(z, t) y^{*}(t) d t, \quad z \in[a, x]
$$

So, by using equation (8) and (4), we take 


$$
\begin{aligned}
y^{*^{\prime}}(z) & =\varphi^{\prime}(z)+g(z, z) y^{*}(z) \\
& +\int_{a}^{z} p(z, t) y^{*}(t) d t, \quad z \in[a, x]
\end{aligned}
$$

Hence

$$
y^{*^{\prime}}(z)=\varphi^{\prime}(z)+\frac{d}{d z} \int_{a}^{z} g(z, t) y^{*}(t) d t, \quad z \in[a, x] \text {. }
$$

By integrating both sides of above equation on $[a, x]$ and conditions (6), we obtain the equation (7). $\square$

Now, let $x \in[a, b]$ be an arbitrary given number and define the following optimal control problem:

Minimize

$$
\int_{a}^{x}\left|\varphi^{\prime}(t)+g(t, t) y(t)+v(t)-u_{1}(t)\right| d t
$$

subject to

$$
\begin{aligned}
& y^{\prime}(t)=u_{1}(t), \quad t \in[a, x] \\
& v^{\prime}(t)=u_{2}(t), \quad t \in[a, x] \\
& r\left(t, y, u_{2}\right)=0, \quad t \in[a, x] \\
& y(a)=\varphi(a), v(a)=0,
\end{aligned}
$$

where $(y(),. v()$.$) and \left(u_{1}(),. u_{2}().\right)$ are state and control variables, respectively, and for $t \in[a, x]$

$$
r\left(t, y, u_{2}\right)=\int_{a}^{t}\left(u_{2}(w)-p(t, w) y(w)\right) d w .
$$

Theorem II.2: Let $x \in[a, b]$ be an arbitrary given number and pairs $\left(y^{*}(),. v^{*}().\right)$ and $\left(u_{1}^{*}(),. u_{2}{ }^{*}().\right)$ be optimal state and control of the problem (9), respectively. Then function $y^{*}($.$) satisfies equation (7).$

Proof: Assume that $x \in[a, b]$ is an arbitrary given number. By theorem II.1, it is sufficient that we show pair $\left(y^{*}(),. v^{*}().\right)$ is the solution of problem (4)-(6). Define the following problem corresponding to the problem (9):

\section{Minimize}

$$
J(y, v)=\int_{a}^{x}\left|\varphi^{\prime}(t)+g(t, t) y(t)+v(t)-y^{\prime}(t)\right| d t
$$

subject to

$$
\begin{aligned}
& \int_{a}^{t}\left(v^{\prime}(w)-p(t, w) y(w)\right) d w=0, \quad t \in[a, x] \\
& y(a)=\varphi(a), v(a)=0 .
\end{aligned}
$$

Now, let $y()=.\bar{y}($.$) be the solution of the equation (1),$ and $\quad v()=.\bar{v}($.$) satisfies equation (12) where$ $y()=.\bar{y}($.$) . It is trivial that pair \left(y^{*}(),. v^{*}().\right)$ satisfies initial conditions (13) and $J(\bar{y}, \bar{v})=0$. On other hand, since $\left(y^{*}(),. v^{*}().\right)$ and $\left(u_{1}^{*}(),. u_{2}^{*}().\right)$ are optimal solutions of problem (9), pair $\left(y^{*}(),. v^{*}().\right)$ is the optimal solution of the problem (11)-(13) and we have $J\left(y^{*}, v^{*}\right)=0$. Thus

$$
\int_{a}^{x}\left|\varphi^{\prime}(z)+g(z, z) y^{*}(z)+v^{*}(z)-y^{\prime^{*}}(z)\right| d t=0 .
$$

Hence

$$
y^{\prime^{*}}(t)=\varphi^{\prime}(t)+g(t, t) y^{*}(t)+v^{*}(t) .
$$

Moreover, equation (5) and initial conditions (6) hold for pair $\left(y^{*}(),. v^{*}().\right)$. Thus, pair $\left(y^{*}(),. v^{*}().\right)$ is the solution of the problem (4)-(6) and this completes proof. $\square$

Now let $n \in \mathrm{N}$ be a given large number. Set $x_{0}=a$, $h=\frac{b-a}{n}$ and $x_{j}=a+j h$ for $j=1,2, \ldots, n$. The corresponding optimal control problem (9) for $x=x_{j}$, $j=1,2, \ldots, n$ is as follows:

Minimize

$$
\int_{a}^{x_{j}}\left|\varphi^{\prime}(t)+g(t, t) y(t)+v(t)-u_{1}(t)\right| d t
$$

subject to

$$
\begin{aligned}
& y^{\prime}(t)=u_{1}(t), \quad t \in\left[a, x_{j}\right] \\
& v^{\prime}(t)=u_{2}(t), \quad t \in\left[a, x_{j}\right] \\
& r\left(t, y, u_{2}\right)=0, \quad t \in\left[a, x_{j}\right] \\
& y(a)=\varphi(a), v(a)=0,
\end{aligned}
$$

where $r(., .,$.$) is defined by (10). We rewrite problem (14)$ for $j=1,2, \ldots, n$ as follows:

\section{Minimize}

$$
\sum_{k=1}^{j} \int_{x_{k-1}}^{x_{k}}\left|\varphi^{\prime}(t)+g(t, t) y(t)+v(t)-u_{1}(t)\right| d t
$$

subject to

$$
\begin{aligned}
& y^{\prime}(t)=u_{1}(t), \quad t \in\left[x_{k-1}, x_{k}\right], k=1,2, \ldots, j \\
& v^{\prime}(t)=u_{2}(t), \quad t \in\left[x_{k-1}, x_{k}\right], k=1,2, \ldots, j \\
& r\left(t, y, u_{2}\right)=0, \quad t \in\left[x_{k-1}, x_{k}\right], k=1,2, \ldots, j \\
& y\left(x_{0}\right)=\varphi\left(x_{0}\right), v\left(x_{0}\right)=0 .
\end{aligned}
$$

By assumption

$$
\mu(t)=\varphi^{\prime}(t)+g(t, t) y(t)+v(t)-u_{1}(t), t \in[a, b]
$$

the problems (15) for all $j=1,2, \ldots, n$ is equivalent to the following multi-objective programming problem:

Minimize

$\left\{\int_{x_{0}}^{x_{1}}|\mu(z)| d z, \int_{x_{0}}^{x_{1}}|\mu(z)| d z+\int_{x_{1}}^{x_{2}}|\mu(z)| d z\right.$,

$$
\left.\ldots, \int_{x_{0}}^{x_{1}}|\mu(z)| d z+\int_{x_{1}}^{x_{2}}|\mu(z)| d z+\ldots+\int_{x_{n-1}}^{x_{n}}|\mu(z)| d z\right\}
$$

subject to $\quad y^{\prime}(t)=u_{1}(t), \quad t \in\left[x_{k-1}, x_{k}\right]$,

$$
\begin{aligned}
& v^{\prime}(t)=u_{2}(t), \quad t \in\left[x_{k-1}, x_{k}\right], \\
& r\left(t, y, u_{2}\right)=0, \quad t \in\left[x_{k-1}, x_{k}\right], \\
& y\left(x_{0}\right)=\varphi\left(x_{0}\right), v\left(x_{0}\right)=0, \\
& k=1,2, \ldots, j \quad, j=1,2, \ldots, n .
\end{aligned}
$$

By multi-objective programming methods (see [43]), we 
can use the following problem instead of problem (16): Minimize

$$
J=\sum_{k=1}^{n}(n-k+1) \int_{x_{k-1}}^{x_{k}}|\mu(z)| d z
$$

subject to

$$
\begin{aligned}
& y^{\prime}(t)=u_{1}(t), \quad t \in\left[x_{k-1}, x_{k}\right], k=1,2, \ldots, n \\
& v^{\prime}(t)=u_{2}(t), \quad t \in\left[x_{k-1}, x_{k}\right], k=1,2, \ldots, n, \\
& r\left(t, y, u_{2}\right)=0, \quad t \in\left[x_{k-1}, x_{k}\right], k=1,2, \ldots, n \\
& y\left(x_{0}\right)=\varphi\left(x_{0}\right), v\left(x_{0}\right)=0 .
\end{aligned}
$$

Define the following problem for $k=1,2, \ldots, n$ :

Minimize

$$
J_{k}=(n-k+1) \int_{x_{k-1}}^{x_{k}}|\mu(z)| d z
$$

subject to

$$
\begin{aligned}
& y^{\prime}(t)=u_{1}(t), \quad t \in\left[x_{k-1}, x_{k}\right], \\
& v^{\prime}(t)=u_{2}(t), \quad t \in\left[x_{k-1}, x_{k}\right], \\
& r_{k-1}\left(t, y, u_{2}\right)=0, \quad t \in\left[x_{k-1}, x_{k}\right], \\
& y\left(x_{k-1}\right)=y^{[k-1]}, v\left(x_{k}\right)=v^{[k-1]} .
\end{aligned}
$$

where $y^{[k-1]}$ and $v^{[k-1]}$ are known numbers which we can obtain these numbers by solving problem (22)-(26) on interval $\left[x_{k-2}, x_{k-1}\right]$. Moreover, for $t \in\left[x_{k-1}, x_{k}\right]$

$$
r_{k-1}\left(t, y, u_{2}\right)=\int_{x_{k-1}}^{t}\left(u_{2}(w)-p(t, w) y(w)\right) d w .
$$

Now we have the following theorem:

Theorem II.3: Let $\quad\left(y_{(k-1)}^{*}(),. v_{(k-1)}^{*}().\right)$ and $\left(u_{(k-1), 1}^{*}(),. u_{(k-1), 2}^{*}().\right)$ for any $k=1,2, \ldots, n$ be optimal state and control of problem (22)-(26), respectively. Then optimal solutions of problem (17)-(21) are as follows:

$$
\left\{\begin{array}{l}
y^{*}(t)=y_{(k-1)}^{*}(t), \quad t \in\left[x_{k-1}, x_{k}\right], k=1,2, \ldots, n \\
v^{*}(t)=v_{(k-1)}^{*}(t), \quad t \in\left[x_{k-1}, x_{k}\right], k=1,2, \ldots, n \\
u_{1}^{*}(t)=u_{(k-1), 1}^{*}(t), \quad t \in\left[x_{k-1}, x_{k}\right], k=1,2, \ldots, n \\
u_{2}^{*}(t)=u_{(k-1), 2}^{*}(t), \quad t \in\left[x_{k-1}, x_{k}\right], k=1,2, \ldots, n .
\end{array}\right.
$$

Proof: Consider the following sets:

$D=\left\{\left(y, v, u_{1}, u_{2}\right):\left(y, v, u_{1}, u_{2}\right)\right.$ satisfies in equations

$$
\text { (18)-(20) on } \left.\left[x_{0}, x_{n}\right]\right\} \text {, }
$$

$D_{k}=\left\{\left(y, v, u_{1}, u_{2}\right):\left(y, v, u_{1}, u_{2}\right)\right.$ satisfies in equations

$$
\text { (23)-(26) on } \left.\left[x_{k-1}, x_{k}\right]\right\}, \quad k=1,2, \ldots, n
$$

It is trivial that $D=\bigcup_{k=1}^{n} D_{k}$. In addition, the value of objective functional in problems (17)-(21) and (22)-(26) are nonnegative. Thus, we have:

$$
\underset{\left(y, v, u_{1}, u_{2}\right) \in D}{\operatorname{Minimize}} J=\sum_{k=1}^{n}\left(\underset{\left(y, v, u_{1}, u_{2}\right) \in D_{k}}{\operatorname{Minimize}} J_{k}\right)
$$

The system (27) is immediate from latter relation and optimality principle in continuous-time dynamic programming which initiated by Bellman (see section 8 of [44]).

Remark II-4: Note that $y^{[0]}=\varphi\left(x_{0}\right)$ and $v^{[0]}=0$.

\section{Linear programming problems}

In this section, we convert the continuous problem (22)(28) to an equivalent discrete problem and solve the obtained problem by linear programming method (see [45]). For this purpose, consider the following approximations in numerical differentiation and integration for $k=1,2, \ldots, n$ and $t=x_{k-1}, x_{k}$ :

$$
\begin{aligned}
& \int_{x_{k-1}}^{x_{k}}|\mu(z)| d z \approx \frac{h}{2}\left(\left|\mu\left(x_{k-1}\right)\right|+\left|\mu\left(x_{k}\right)\right|\right) \\
& y^{\prime}(t) \approx \frac{1}{h}\left(y\left(x_{k}\right)-y\left(x_{k-1}\right)\right), \quad v^{\prime}(t) \approx \frac{1}{h}\left(v\left(x_{k}\right)-v\left(x_{k-1}\right)\right), \\
& \int_{x_{k-1}}^{x_{k}}\left(u_{2}(w)-p\left(x_{k}, w\right) y(w)\right) d w \approx \\
& \frac{h}{2}\left(u_{2, k-1}-p\left(x_{k}, x_{k-1}\right) y_{k-1}+u_{2, k}-p\left(x_{k}, x_{k}\right) y_{k}\right) .
\end{aligned}
$$

Using the above approximation we can transform problem (22)-(28) to the following corresponding problem for $k=1,2, \ldots, n$ :

\section{Minimize}

$$
J_{k}=\frac{h}{2}(n-k+1)\left(\left|\mu_{k-1}\right|+\left|\mu_{k}\right|\right)
$$

subject to

$$
\begin{aligned}
& y_{k}-y_{k-1}-h u_{1, k-1}=0, \quad y_{k}-y_{k-1}-h u_{1, k}=0, \\
& v_{k}-v_{k-1}-h u_{2, k-1}=0, \quad v_{k}-v_{k-1}-h u_{2, k}=0, \\
& u_{2, k-1}-p\left(x_{k}, x_{k-1}\right) y_{k-1}+u_{2, k}-p\left(x_{k}, x_{k}\right) y_{k}=0, \\
& y_{k-1}=y^{[k-1]}, v_{k-1}=v^{[k-1]} .
\end{aligned}
$$

where for $l=k-1, k$ we have:

$$
\begin{gathered}
u_{1, l}=u_{1}\left(x_{l}\right), u_{2, l}=u_{2}\left(x_{l}\right), \\
\mu_{l}=\mu\left(x_{l}\right), y_{l}=y\left(x_{l}\right), v_{l}=v\left(x_{l}\right) .
\end{gathered}
$$

Now by applying the techniques of linear and nonlinear programming $[45,46]$, and relation

$$
\mu(t)=\varphi^{\prime}(t)+g(t, t) y(t)+v(t)-u_{1}(t), \quad t \in[a, b]
$$

the nonlinear problem (28) be converted to the following corresponding linear programming problem for $k=1,2, \ldots, n$ :

Minimize $\quad J_{k}=\frac{h}{2}(n-k+1)\left(z_{k-1}+z_{k}\right)$

subject to

$$
\begin{aligned}
& \mu_{k-1}+z_{k-1} \leq 0,-\mu_{k-1}+z_{k-1} \leq 0, \\
& \mu_{k}+z_{k} \leq 0,-\mu_{k}+z_{k} \leq 0, \\
& g\left(x_{k-1}, x_{k-1}\right) y_{k-1}+v_{k-1}-u_{1, k-1}-\mu_{k-1}=-\varphi^{\prime}\left(x_{k-1}\right), \\
& g\left(x_{k}, x_{k}\right) y_{k}+v_{k}-u_{1, k}-\mu_{k}=-\varphi^{\prime}\left(x_{k}\right), \\
& y_{k}-y_{k-1}-h u_{1, k-1}=0, y_{k}-y_{k-1}-h u_{1, k}=0,
\end{aligned}
$$




$$
\begin{aligned}
& v_{k}-v_{k-1}-h u_{2, k-1}=0, v_{k}-v_{k-1}-h u_{2, k}=0, \\
& u_{2, k-1}-p\left(x_{k}, x_{k-1}\right) y_{k-1}+u_{2, k}-p\left(x_{k}, x_{k}\right) y_{k}=0, \\
& y_{k-1}=y^{[k-1]}, v_{k-1}=v^{[k-1]}, z_{k-1} \geq 0, z_{k} \geq 0,
\end{aligned}
$$

where decision variables of this problem are $z_{l}, \mu_{l}, y_{l}, v_{l}, u_{1, l}$ and $u_{2, l}$ for $l=k-1, k$. Not that by solving problems (29), we obtain the approximations $y_{k}^{*}$, $k=1,2, \ldots n$ for exact solution of problem (1), means $y^{*}\left(x_{k}\right), k=1,2, \ldots, n$.

Remark III.1: Note that, in this approach, we solve the linear programming problem (29) and use theorem 2-3 to approximate the solution of equation (1).

In next section, we illustrative the efficiency of our approach in some numerical examples.

\section{Simulation results}

In this section, we use our approach to solve two linear Volterra integral equations. Here we apply the MATLAB software and simplex method [45] for solving linear programming problem (29).

Example IV.1: Consider the following Volterra integral equation of second kind:

$$
\left\{\begin{array}{l}
y(x)=e^{x} \sin (x)+\int_{0}^{x} \frac{2+\cos (x)}{2+\cos (t)} y(t) d t, \\
x \in[0,1] .
\end{array}\right.
$$

The exact solution of equation (34) is

$$
\begin{aligned}
y(x) & =e^{x} \sin (x) \\
& +e^{x}(2+\cos (x))(\ln (3)-\ln (2+\cos (x))),
\end{aligned}
$$

Here for $n=10,20,50$ and $n=100$, we solve corresponding problem (29) for equation (30). In Figures $1-4$, the connected line indicates the graph of exact solutions. The comparis on of obtained results and exact solution for equation (29) are showed in Table 1.

Example IV.2: Consider the following Volterra integral equation of second kind:

$$
\left\{\begin{array}{l}
y(x)=\sin (x)+\int_{0}^{x} 2 \cos (x-t) y(t) d t, \\
x \in[0,1] .
\end{array}\right.
$$

Here for $n=10,20,30$ and $n=40$, the corresponding problem (29) for equation (31) is solved. The connected line indicates the graph of exact solutions. We can compare the obtained results and exact solution of equation (31) in Table 2.

\section{Conclusions}

In this paper, we obtained optimal control problems corresponding Volterra integral equation. By discretization method these optimal control problems converted to the corresponding linear programming problems. Thus we can solve linear programming problems instead of Volterra integral equations. By this approach, we can obtain a good approximation for the solution of linear Volterra integral equation.

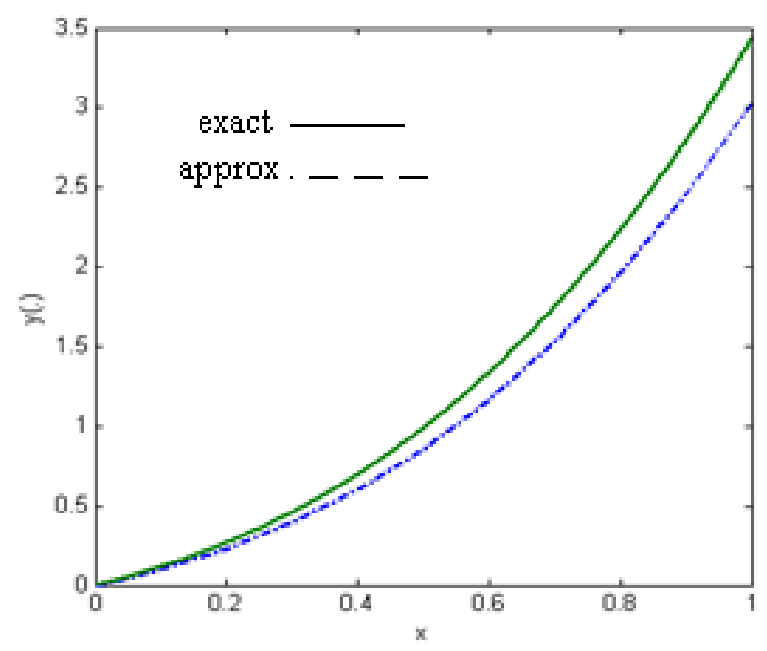

Fig. 1: The approximate solution for $n=10$

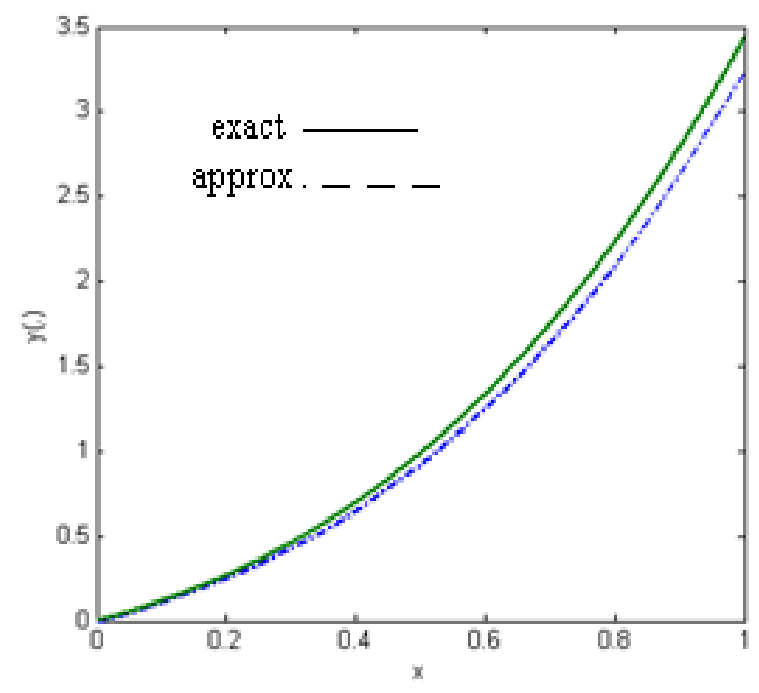

Fig. 2: The approximate solution for $n=20$

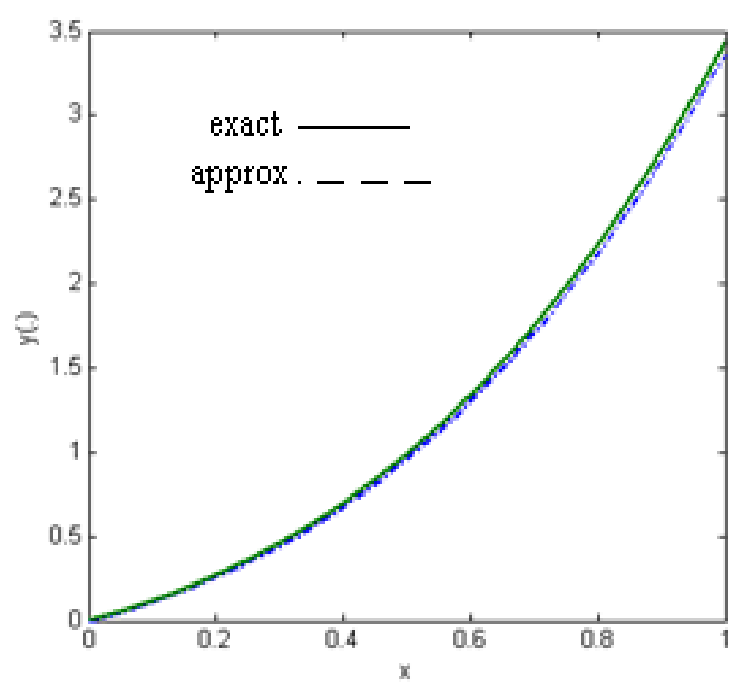

Fig .3: The approximate solution for $n=50$ 


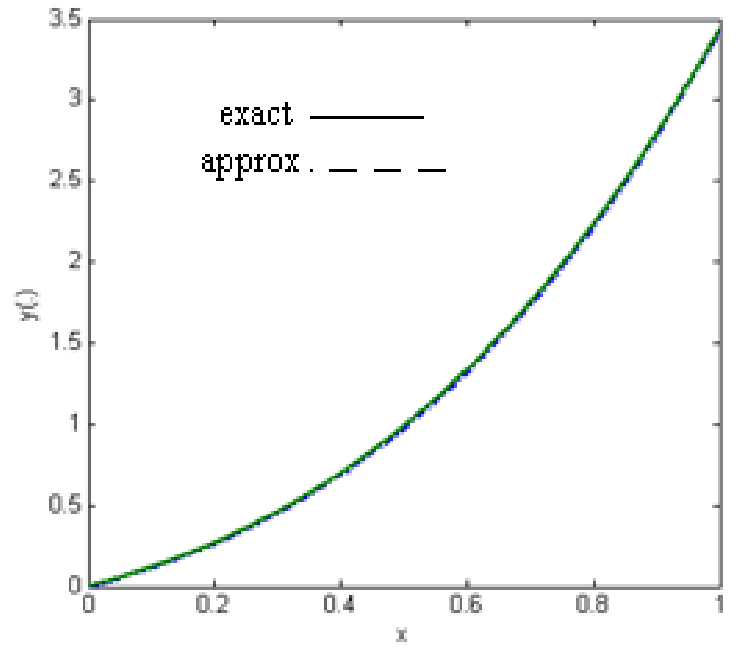

Fig. 4: The approximate solution for $n=100$

Table 1: Comparis on of exact and approximate solutions for Ex. IV.1 .

\begin{tabular}{|c|c|c|c|c|l|}
\hline$x$ & $\begin{array}{c}\text { Approximate } \\
\text { solution for } \\
n=10\end{array}$ & $\begin{array}{c}\text { Approximate } \\
\text { solution for } \\
n=20\end{array}$ & $\begin{array}{c}\text { Approximate } \\
\text { solution for } \\
n=50\end{array}$ & $\begin{array}{c}\text { Approximate } \\
\text { solution for } \\
n=100\end{array}$ & $\begin{array}{l}\text { Exact } \\
\text { solution }\end{array}$ \\
\hline$x=0.2$ & 0.23098159 & 0.24826681 & 0.25929638 & 0.26309086 & 0.26692094 \\
\hline$x=0.5$ & 0.86113061 & 0.92262360 & 0.96197727 & 0.97553724 & 0.98809616 \\
\hline$x=0.8$ & 1.96419901 & 2.09898018 & 2.18542479 & 2.21524535 & 2.21524535 \\
\hline
\end{tabular}

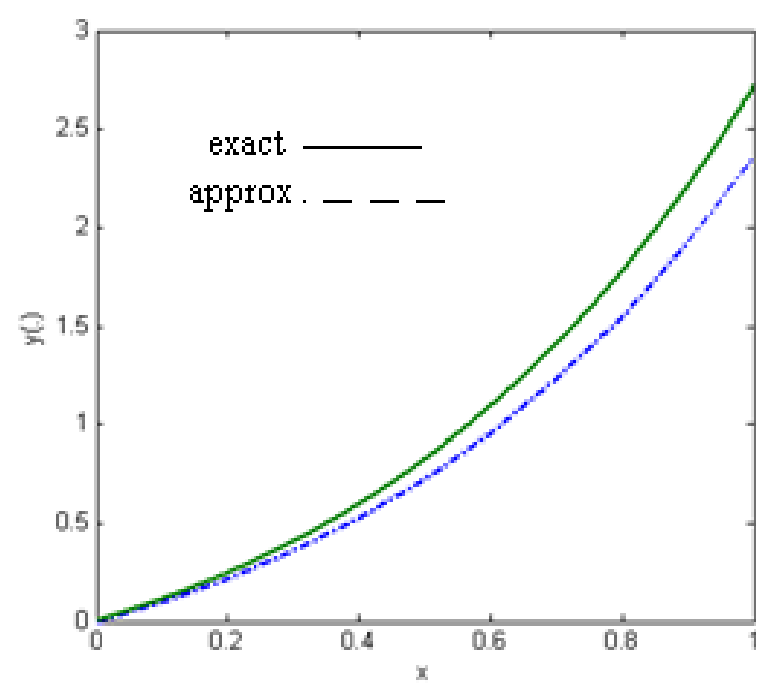

Fig. 5: The approximate solution for $n=10$.

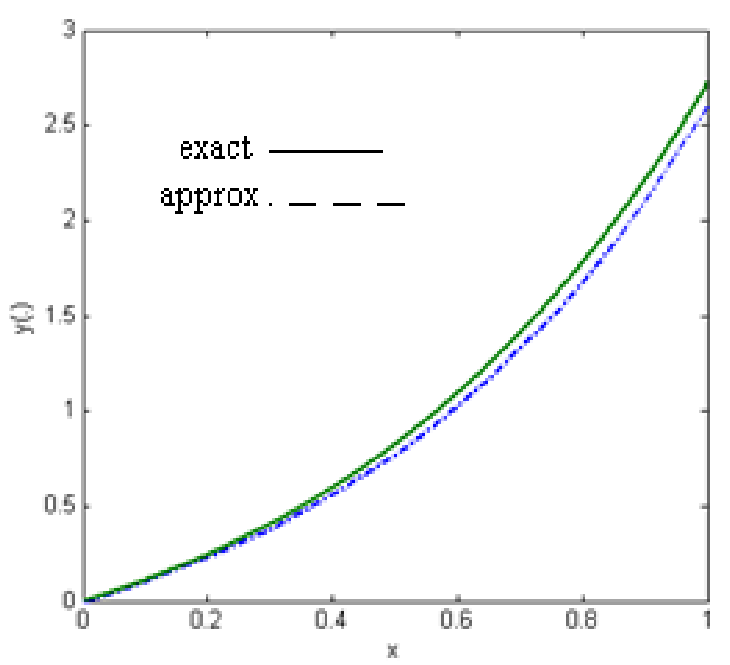

Fig. 6: The approximate solution for $n=20$.

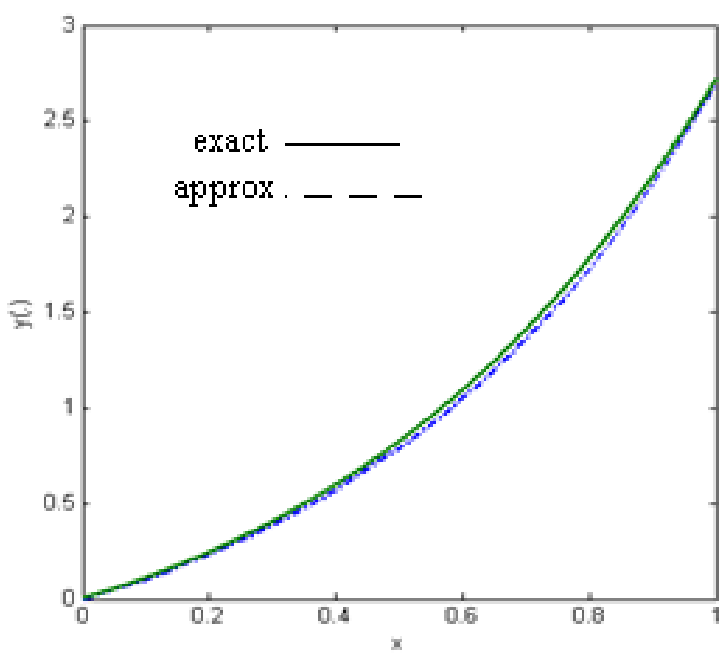

Fig. 7: The approximate solution for $n=30$

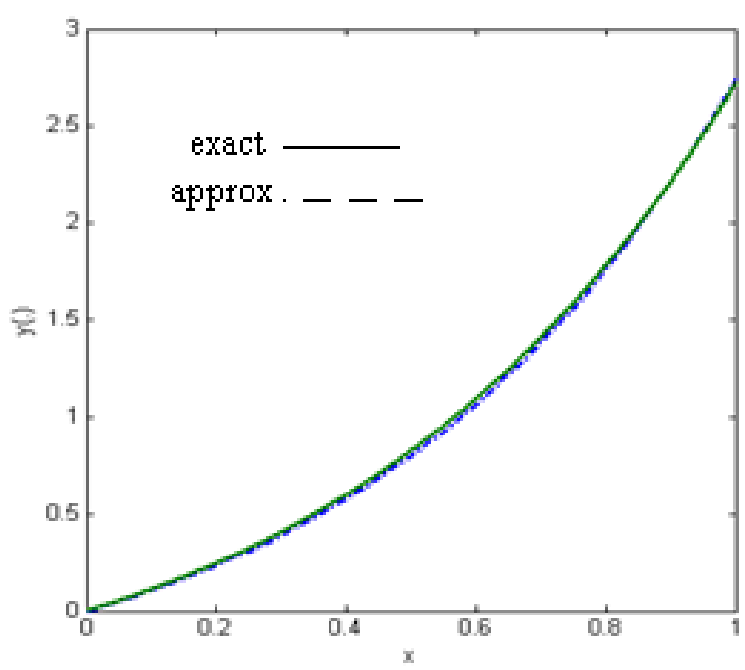

Fig. 8: The approximate solution for $n=40$ 
Table 2: Comparison of exact and approximate solutions for Ex. IV.2

\begin{tabular}{|c|c|c|c|c|c|}
\hline$x$ & $\begin{array}{c}\text { Approximate } \\
\text { solution for } \\
n=10\end{array}$ & $\begin{array}{c}\text { Approximate } \\
\text { solution for } \\
n=20\end{array}$ & $\begin{array}{c}\text { Approximate } \\
\text { solution for } \\
n=30\end{array}$ & $\begin{array}{c}\text { Approximate } \\
\text { solution for } \\
n=40\end{array}$ & $\begin{array}{c}\text { Exact } \\
\text { solution }\end{array}$ \\
\hline$x=0.2$ & 0.21950041 & 0.23111194 & 0.23534042 & 0.23753096 & 0.24428055 \\
\hline$x=0.5$ & 0.72596658 & 0.77435737 & 0.79243764 & 0.80190046 & 0.82436063 \\
\hline$x=0.8$ & 1.54942048 & 1.68180727 & 1.73246743 & 1.75923588 & 1.78043274 \\
\hline
\end{tabular}

\section{REFERENCES}

[1] K. Atkinson, The numerical solution of integral equations of the second kind, Co mberidge University press, 1999.

[2] K. Atkinson and W. Han, Theoretical Numerical Analysis: A Functional Analysis Framework, vol. 39 of Texts in Applied Mathematics, Springer, Dordrecht, The Netherlands, 3rd edition, 2009.

[3] C. T. H. Baker, The Numerical Treatment of Integral Equations, Monographs on Numerical Analysis, Clarendon Press, Oxford, UK, 1977.

[4] A. J. Jerri, Introduction to integral equations with applications, Wiley, London, 1999.

[5] A. M. Wazwaz, A First Course in Integral Equations, WSPC, New Jersey, USA 1997.

[6] E. Babolian and A. Davary, Numerical Implementation of Adomian Decomposition Method for Linear Volterra Integral Equations for the Second Kind., Applied Mathematics and Computation, Vol. 165, pp. 223-227, 2005.

[7] W. F. Blyth, R.L. May, Widyaningsih, P., .The Solution of Integral Equations Using Walsh Functions and a Multigrid Approach, In J. Noye et a l., Editors, Computational Techniques and Applications: CTAC97. Proceedings, 99-106. World Scientific, Singapore, 1998.

[8] W.F. Blyth, R.L. May and P. Widyaningsih, Volterra Integral Equations Solved in Fredholm Form Using Walsh Functions, Anziam J, Vol. 45 (E), pp. 269-282, 2004.

[9] S. Bhattacharya and B. N. Mandal, Use of Bernstein Polynomials in Numerical Solutions of Volterra Integral Equations, Applied Mathematical Sciences, Vol. 2, no. 36, 1773 - 1787, 2008.

[10] J. G Blom, H. Brunner, Jun, Algorithm 689: Discretized collocation and iterated collocation for nonlinear Volterra integral equations of the second kind. ACM Trans. Math. Software, Vol. 17, no. 2, pp. 167-177, 1991.

[11] H. Brunner, A. Makroglou and R.K. Miller, Mixed Interpolation Collocation Methods for first and Second Volterra Integro-Differential Equations with
Periodic Solution., Appl. Numer. Math, Vol. 23, pp. 381-402, 1997.

[12] H. Brunner, Collocation Methods for Volterra Integral and Related Functional Equations Methods, Cambridge University Press 2004.

[13] H. Brunner, The numerical solution of weakly singular Volterra integral equations by collocation on graded meshes. Math. Comp. Vol. 45, no. 172, pp. 417-437, 1985.

[14] H. Brunner, Polynomial spline collocation methods for Volterra integro-differential equations with weakly singular kernels. IMA J. Numer. Anal. Vol. 6, no. 2, pp. 221-239, 1986.

[15] T. Tang, A note on collocation methods for Volterra integro-differential equations with weakly singular kernels. IMA J. Numer. Anal, Vol. 13, no. 1, pp. 9399, 1993.

[16] E. Hairer, C. Lubich and M. Schlichte, Fast numerical solution of nonlinear Volterra convolution equations. SIAM J, Sci. Stat. Comput. Vol. 6, no. 3, pp. 531-541, 1985.

[17] K. Maleknejad and N. Aghazadeh, "Numerical solution of Volterra integral equations of the second kind with convolution kernel by using Taylor-series expansion method," Applied Mathematics and Computation, Vol. 161, no. 3, pp. 915-922, 2005.

[18] Y. Ren, B. Zhang and H. Qiao, A simple Taylorseries expansion method for a class of second kind integral equations, J. Comput. Appl. Math. Vol. 110, pp 15-24, 1999.

[19] A. M, Wazwaz, Two Methods for Solving Integral Equations., Appl. Math. Comput., 77, 1996, pp. 79.89.

[20] E. Deeba, S. A. Khuri and S. Xie, An Algorithm for Solving a Nonlinear Integro-Differential Equation., Appl. Math. Comput., Vol. 115, pp. 123.131, 2000.

[21] C. Canuto, M.Y. Hussaini, A. Quarteroni and T.A. Zang, Spectral Methods: Fundamentals in Single Domains, Springer-Verlag 2006.

[22] L. M. Delves and J. L. Mohanmed, Computational Methods for Integral Equations, Cambridge University Press, 1985.

[23] G.N. Elnagar and M. Kazemi, Chebyshev spectral solution of nonlinear Volterra-Hammerstein integral equations, J. Comput. Appl. Math., Vol. 76, pp. 147158, 1999.

[24] J. S. Hesthaven, S. Gottlieb and D. Gottlieb, Spectral Methods for Time-Dependent Problems. No. 21 in Cambridge Monographs on Applied and Computational Mathematics. Cambridge University Press, Cambridge UK. 2007.

[25] J. Shen and T. Tang, Spectral and High-Order Methods with Applications, Science Press, Beijing, 2006.

[26] T. Tang, X. Xu and J. Cheng, On spectral methods for Volterra type integral equations and the convergence analysis. J. Comp. Math. Vol. 26, no. 6 , pp. 825-837, 2008.

[27] H. C. Tian, Spectral Method for Volterra Integral Equation, MSc Thesis, Simon Fraser University 1995. 
[28] D. M. Bedivan and G. J. Fix, Analysis of finite element approximation and quadrature of Volterra integral equations. Numer. Methods Partial Differential Equations vol. 13, no. 6, pp. 663-672. 1997.

[29] M. Muhammad, A. Nurmuhammad, M. Mori, and M. Sugihara, Nu merical solution of integral equations by means of the sinc collocation method based on the double exponential transformation. J. Comput. Appl. Math. Vol. 177, pp. 269-286, 2005.

[30] J. Rashidinia and M. Zarebnia, New approach for numerical solution of Volterra integral equations of the second kind, IUST International Journal of Engineering Science, Vol. 19, No.5-2, pp. 59-65, 2008.

[31] H. Brunner, Lin and Y., Zhang, S. Higher accuracy methods for second-kind Volterra integral equations based on asymptotic expansions of iterated Galerkin methods. J. Integral Equations Appl. Vol. 10, no. 4, pp. 375-396, 1998.

[32] Z. Wan, Y. Chen, and Y.Huang, "Legendre spectral Galerkin method for second-kind Volterra integral equations," Frontiers of Mathematics in China, vol. 4, no. 1, pp. 181-193, 2009.

[33] K. Maleknejad, M. Tavassoli Kajani and Y. Mahmoudi, Numerical solution of linear Fredholm and volterra integral equation of the second kind by using Legendre wavelets, Kybernetes, Vol. 32, No. 9/10, pp. 1530-1539, 2003.

[34] J. Saberi-nadjafi and M. Heidari, A generalized block-by-block method for solving linear Volterra integral equations, Applied Mathematics and Computation, Vol. 188, pp. 1969-1974, 2007.

[35] S. Abbasbandy, "Application of He's homotopy perturbation method to functional integral equations," Chaos, Solitons and Fractals. Vol. 31, pp. 1243-1247, 2007.

[36] S. Abbasbandy, Numerical solution of integral equation: Homotopy perturbation method and Adomian's decomposition method, Appl. Math. Comput. Vol.173, pp. 493-500, 2006.

[37] A. Adawi, F. Awawdeh and H. Jaradat, A Numerical Method for Solving Linear Integral Equations, Int. J. Contemp. Math. Sciences, Vol. 4, no. 10, pp. $485-$ 496, 2009.

[38] J. Biazar and Z. Ayati, A Maple Program for the Second Kind of Volterra Integral Equations by Homotopy perturbation Method, International Mathematical Forum, Vol. 5, no. 67, pp. 3323 - 3326, 2010.

[39] S.J. Liao, Beyond Perturbation: Introduction to the Homotopy Analysis Method, Chapman \& Hall/CRC Press, Boca Raton, 2003.

[40] J. T. Betts, Practical Methods for Optimal Control and Estimation Using Nonlinear Programming, Siam, Philadelphia, second edition, 2010.

[41] D. Burghes and A. Graham, Introduction to control theory, including optimal control, Ellis Horwood, 1980.
[42] E. R. Pinch, Optimal control and calculus of variations, Paperback ed. Oxford; New York: Oxford Univercity Press, 1995.

[43] D. Jones, M. Tamiz and J. Ries, New development in multiple objective and goal programming, SpringerVerlag Berlin Hiedelberg, 2010.

[44] C. K. Chui and G.Chen, Linear systems and optimal control, Springer, 1988.

[45] M. S. Bazaraa, J. J. Javis, H. D. Sheralli, linear programming, New York; published by Wiley \& Sons, 1990.

[46] M. S. Bazaraa, H. D. Sheralli, C. M. Shetty, Nonlinear programming: Theory and Application, New York; published by Wiley \& Sons, 2006.

How to cite this paper: Sohrab Effati,Mohammad Hadi Noori Skandari,"Optimal Control Approach for Solving Linear Volterra Integral Equations", International Journal of Intelligent Systems and Applications(IJISA), vol.4, no.4, pp.40-46, 2012. DOI: $10.5815 /$ ijisa.2012.04.06 